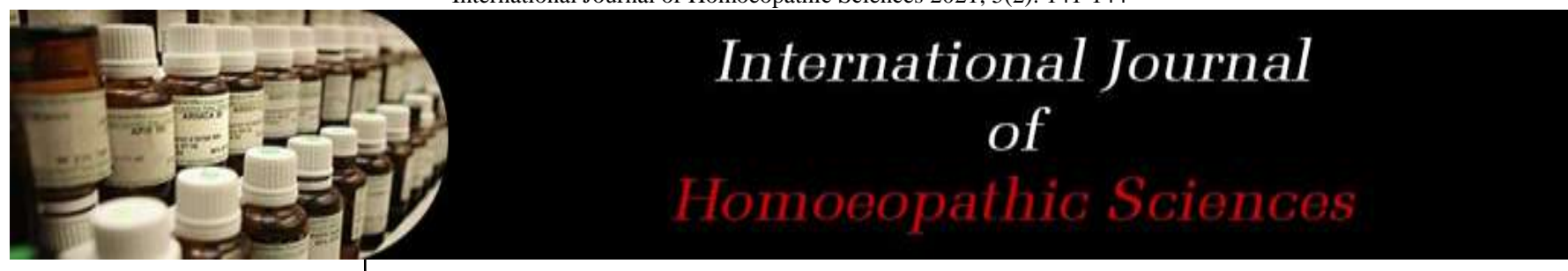

E-ISSN: 2616-4493 P-ISSN: 2616-4485 www.homoeopathicjournal.com IJHS 2021; 5(2): 141-144 Received: 02-01-2021 Accepted: 06-02-2021

Ourooj Safi

Independent Researcher, Al Ain, United Arab Emirates
Corresponding Author: Ourooj Safi

Independent Researcher, Al Ain, United Arab Emirates

\section{Respiratory pandemics and homoeopathy}

\section{Ourooj Safi}

DOI: $\underline{\text { https://doi.org/10.33545/26164485.2021.v5.i2c.373 }}$

\section{Abstract}

The Coronavirus Pandemic has created so much devastation in such a short time. This virus has taken so many lives and shattered the economy of World. In such a panic situation when there is no medicine available Homoeopathic philosophy can cure the disease. In this paper I discussed about the role of homoeopathy in various past Respiratory Pandemics. The article shows how homoeopathy can play effective role in prevention and cure of this disease. It is the correct time to give homoeopathic medicine a chance to save lives and being cheap these medicines are not putting any financial burden. People can get assured and inexpensive treatment. Homoeopathic medicines along with vaccination, face mask and social distancing can give relief in the coronavirus Pandemic.

Keywords: Respiratory pandemics, homoeopathy, Coronavirus Pandemic

\section{Introduction}

Whole world is going through the devastating effect of corona virus. In December 2019, a breakout of a strange disease was marked in Wuhan Hubei China ${ }^{[1]}$. It was having symptoms of pneumonia that is dry cough, fever and tiredness and occasional diarrhea. With only 41 patients starting the disease it took only few weeks to spread throughout the Wuhan region of China and from there to the rest of the world. Its spread shows its highly contagious nature. World health organization declared it Pandemic on 11 March 2020. The outbreak of the COVID-19 has been spread nearly in all the countries of the world and has kept people's health at high risk. The disease is named COVID 19 because it belongs to the SARS coronavirus, the virus causes the disease severe acute respiratory syndrome coronavirus 2 , or SARS-CoV-2, referring to the symptoms of the disease and the virus that spreads it. The transmission of the Severe Acute Respiratory Syndrome coronavirus 2 (SARS-CoV-2) take place during the asymptomatic phase of the disease. Its incubation periods vary from 2 days to 14 days. There is no specific treatment is available for this disease, but proper management and precautions may give relief.

\section{COVID 19}

This virus is a single-stranded positive-sense RNA virus that causes infection and respiratory failure ${ }^{[2]}$. The genome of this virus is spotted and is very much similar tothe SARS$\mathrm{CoV}\left(80 \%\right.$ similarity) and MERS-CoV (50\% similarity) ${ }^{[3]}$. Source of this virus are some animals. The virus was having $86.9 \%$ resemblance to a bat coronavirus, so it is assumed to developed from bats ${ }^{[4]}$. The mode of transmitting the virus is through Droplets, infected hands, skin-to-skin contact, as well as contaminated surface ${ }^{[5]}$. It is a human to human as well as surface to human transmission ${ }^{[6]}$. The incubation period of the disease is between 214 days with 5.2 days as an average incubation period ${ }^{[7]}$. The symptoms of COVID 19varies from mild to severe which aresore throat, dry cough, sneezing, fatigue, muscle pain, high fever, pneumonia, organ failure and in some cases death ${ }^{[8]}$. This disease puts children, old age and persons with heart disease, diabetes at high risk.

\section{Homoeopathy}

Homoeopathy is based on the law of similia that means like cure like which means the medicines in the crude form produces the same symptoms of disease which is going to cure in potentised form. Homoeopathic medicine intensify normal healing of the body and selfregulatory processes. It restoresthe body's own healing response to disease, using specially prepared, highly diluted preparation. It believes that there is no specific disease effecting part of a person, but it is the whole individual who is affected or sick ${ }^{[9]}$. 
Medicines are considered safe with no adverse reaction and are prepared from plants, animals and minerals. Homoeopathy has played a contributory role in the treatment and prevention in various epidemics in the past.

\section{Homoeopathy in Epidemics}

Right from the beginning homoeopathy has been successful in treating epidemic diseases. The short writing published in Hufeland's journal with the title 'Some type of continuous and remitting fever' Dr Hahnemann wrote how Ignatia Amara was used to cure repeated sporadic fever in children in the year 1797. It was having main symptoms of continuous chills, laxity, cold sweat on forehead and short spasmodic breathing ${ }^{[10]}$. Hahnemann write about the use of Atropa Belladona in the treatment of Scarlet fever epidemic in his short writing "Cure and prevention of scarlet fever which occur in 1799. According to him medicine is best preventive when it can block diseases in its initial stages ${ }^{[11]}$. In 1813 Hahnemann treated Typhus fever epidemic. Cholera epidemic was spread in 1830 in which Dr. Hahnemann recognize the stages of the disease. During this time, the mortality rate from conventional medicines was more than $30-40 \%$ but in the cases under homoeopathic care mortality was less than $10 \%{ }^{[12]}$.

Spanish Flu the Pandemic of 1918 killed around 50 million people and infected one third of the world's population ${ }^{[13]}$. During Pandemic of 1918 role of Homoeopathy was surprising. Dean W.A. Pearson collected 26,795 cases of Influenza treated by Homoeopathic physicians with the mortality of $1.05 \%$ whereas conventional medicines mortality was $30 \%{ }^{[14]}$. Homoeopathy gave the assurance of survival from the Pandemic of 1918. Homoeopaths observed the characteristics symptoms of the disease and treated it accordingly. Arsenic, Bryonia, Eupatorium Perfoliatum, Baptisia, and Gelsemium were the medicines which were used generally during the Spanish flu pandemic, and if the cases were treated during very initial stages than there is less chances of developing Pneumonia which in turn resulted in less mortality ${ }^{[15]}$. Gelsemium was taken as genus epidemicus and it covered near about $95 \%$ of cases ${ }^{[16]}$. Pearson reported on the nurses who were taking care of flu patients were treated at the Hahnemann Hospital. The nurses were working continuously without taking care of their own health, many of them suffered from Influenza but none of them developed Pneumonia due to homoeopathic medicines they have taken ${ }^{[17]}$.

SARS-CoV was emerged in 2003 from Southern China and spread to other countries having 774 deaths and 8098 affected cases ${ }^{[18]}$. In 2012 Middle East Respiratory Syndrome (MERS) was discovered in Saudi Arabia, 2279 patients from 27 countries were infected as reported by the WHO and 876 patients were died with highest mortality rate in Saudi Arabia [19]. SARS was spread to human through cats while MERS was transmitted through camels in human ${ }^{[20]}$. Mortality rate of MERS was $34 \%$ which is higher than the SARS that was $10 \%{ }^{[21]}$. Symptoms of these SARS outbreaks were fever, cough, breathlessness while in severe cases symptoms of Pneumonia, Acute Respiratory Distress Syndrome, Renal Failure, and death were seen [22]. Homoeopathic medicines in this viral infection cases gives a chance to recover. It can also prevent the disease if taken as a preventive. Medicines like Arsenic Alb, Eupatorium Perf, Belladona, Aconite will give satisfactory results. Second line of medicines such as Sulphur, Merc Sol are needed if additional symptoms developed along with the persisting symptoms ${ }^{[23]}$.

In 2009 another pandemic started known as Swine flu or H1NI flu. It was having symptoms of running nose, fever, sneezing, weakness and gastric problem like nausea and vomiting. Homoeopathy had an effective role during the H1N1 Pandemic. A study made by R.T.Mathie shows that the medicine Arsenic Album can prevent and control the pandemic ${ }^{[24]}$. In his survey he collected the data during the peak of pandemic that is from October 2009 to February 2010. Nearly twenty-three Homoeopathic physician helped in data collection. Different symptoms of swine flu were seen in group of 1126 patients but the most common symptoms among them were high temperature, cough and running nose. Based on the symptom's medicines were prescribed to the patients in which Arsenic Alb was prescribed more frequently and it was concluded that Arsenic Alb effectively cure the Swine flu. In India, the Ayush dispensary provided Arsenic Alb. free of cost and was authenticated by the Union Health Ministry for prevention and cure of Swine flu ${ }^{[25]}$.

In June 2012 MERS was identified in the Kingdom of Saudi Arabia where a patient was admitted in hospital with the symptoms of Pneumonia and acute kidney injury ${ }^{[26]}$. Major symptoms were cough, chills, fever, dyspnea, and muscles pain. Serious patients were marked with symptoms such as nausea, vomiting, or diarrhea and in half of these patients' acute kidney damage was reported ${ }^{[27]}$. MERS-CoV is closely associated with coronaviruses ${ }^{[28]}$. Homoeopathy is not popular in Saudi Arab but the study shows that in the early stages of pathology Aconitum Napellus, Arsenicum Album or Eupatorium perfoliatum, Gelsemium, Ipecac works well where as in the later stages Bryonia or Phosphorus are the main drugs, and Antimonium Tartaricum, Baptisia or Camphorin the final stages ${ }^{[28]}$.

The Coronavirus disease 2019 (COVID-19) which is caused by novel coronavirus or severe acute respiratory syndrome. Symptoms of This disease is like other viral upper respiratory illnesses. The disease consists of three main stages: upper respiratory symptoms come under mild stage, non-severe pneumonia, and serious pneumonia complicated by acute respiratory distress syndrome ${ }^{[29]}$. Age and Immune condition of patient plays an important role in the progression of disease ${ }^{[30]}$. It is severe in patients with a history of chronic disease. The virus has strange behavior as initially it behaves like a common virus and progress slowly and on finding the weak immune system it severely affects the lungs causing a very high level of dyspnea. The disease starts with the symptoms like fever, sore throat, cough, muscle pain, loss of smell and taste and progress towards severity with the condition of Pneumonia, Acute renal failure and death.

The guiding signs for findingthe indicated remedyis the totality of symptoms for each patient. The sign and symptoms of COVID 19 observed in large number of patients throughout the world can lead us to select some individualize medicines for different stages of the disease. Repertorisation of totality of symptoms has shown that medicines like Arsenic album, Bryonia, Camphora, Gelsemium, Rhustox works well in this Pandemic. CCRH and Ministry of AYUSH, Govt. of India has announced Arsenic 30 as a useful medicine for the prevention of COVID-19 [31]. Further research shows that Arsenic Album, Camphor, Aconite or Belladonna all were efficient in 
preventing the severe symptoms of COVID-19 and were successful in the complete recovery from the Pandemic disease ${ }^{[32]}$.

Arsenicum Album 30C, a homoeopathicmedicine is prepared by diluting aqueous arsenic trioxide till little or no arsenic remains and used in respiratory disorders. It has been used safely for more than 220 years and had shown positive result in swine flu. Some of the state governments like Rajasthan, Karnataka, Tamil Nadu, Andhra Pradesh and Kerala have recommended Arsenic Alb 30 whereas Mumbai police started taking this for the prevention of disease. $\mathrm{CCRH}$ advised to take itdaily for three days in empty stomach and the medicine should be repeated after one month by following the same schedule till Corona virus infections exist in the area ${ }^{[33]}$.

Another important remedy Camphorasuggested by Doctors Sankaran and Kasariyanseems to be suitable in advanced stage of the disease with lot of prostration, low oxygen concentration, nausea and extreme breathlessness [34]. Camphora $1 \mathrm{M}$ has been used on several patients in Iran and is evidently a coronavirus prophylactic. Camphor $1 \mathrm{M}$ is an immunity enhancer against the COVID 19. Managing director of Bajaj Auto Rajeev Bajaj in an interview to India Today, highlighting its effects. A report says Bajaj Auto set up a homoeopathy center in Pune and about 67,000 bottles of Camphora 1Mwere distributed ${ }^{[35]}$. The medicine was not only distributed among the employees of Bajaj, but the medicine was also distributed among the police force in Pune and Pimpri-Chinchwad and surprisingly no police official has been infected in this region ${ }^{[36]}$.

\section{Conclusion}

Whole world is going through financial pressure because of the health expenditure due to Pandemic and lockdown, lots of funds are spend on the treatment. Many people are dying because of the lack of health facilities and unavailability of resources. People are not reporting cases due to fear of expenditure as the life supporting system and hospital admission bills are high. No reliable medicine is available in Allopathy, though vaccination has developed some hope,but it can also not prevent people from COVID fully. Vaccine can only prevent people from getting seriously sick and may reduce mortality in COVID patients but cannot prevent from getting the infection. From the above discussion it has been proved that Homoeopathy has promising results in Respiratory Pandemics. Based on the law of like cure like it has shown its miraculous affects on patients with no side effects. It is a safe and affordable mode of treatment. The spreading Pandemic can be control by Homoeopathic medicines along with the other protocols like wearing face mask, sanitization and social distancing. The Government can distribute medicine among the people to prevent the disease. Further research and well develop programs in homoeopathic institution along with government strategies can lead to a cheap and assured treatment for the Coronavirus disease. Homoeopathic medicines along with the vaccination can provide an extra protective shield from the disease.

\section{References}

1. Huang C, Wang Y, Li X, Ren L, Zhao J, Hu y, et al. Clinical features of patients infected with 2019 novel coronavirus in Wuhan, China. Lancet 2020;395:427-506. doi:10.1016/S0140-6736(20)30183-

\section{I}

2. Swerdlow DL, Finelli L. Preparation for possible sustained transmission of 2019 novel coronavirus: lessons from previous epidemics. Jama 2020;323;12:1129-1130. 10.1001/jama.2020.1960. [PubMed]

[CrossRef] [Google Scholar]

3. Ren LL, Wang YM, Wu ZQ, Xiang ZC, Guo L, Xu T. Identification of a novel coronavirus causing severe pneumonia in human: a descriptive study. Chinese Med. J 2020;133(9):1015-1024. doi: 10.1097/CM9.0000000000000722 (In Press) [PMC free article] [PubMed] [Google Scholar]

4. Lu R, Zhao X, Li J, Niu P, Yang B, Wu H. Genomic characterization and epidemiology of 2019 novel coronavirus: implications for virus origins and receptor binding. Lancet. 2020;395(10224):565-574. doi: 10.1016/S0140-6736(20)30251-8 [PMC free article] [PubMed] [Google Scholar]

5. Kampf G, Todt D, Pfaender S, Steinmann E. Persistence of coronaviruses on inanimate surfaces and its inactivation with biocidal agents. J Hosp Infect. 2020;104(3):246-251.doi:

10.1016/j.jhin.2020.01.022. [PMC

free article] [PubMed] [CrossRef] [Google Scholar

6. Chan JFW, Yuan S, Kok KH, et al. A familial cluster of pneumonia associated with the 2019 novel coronavirus indicating person-to-person transmission: a study of a family cluster. Lancet. 2020;395(10223):514-523. doi:10.1016/S0140-6736(20)30154-9 (published online Jan 24)

7. Li Q, Guan X, Wu P, Wang X, Zhou L, Tong Y. Early transmission dynamics in Wuhan, China, of novel coronavirus-infected pneumonia. N. Engl. J Med. 2020;382:1199-1207.

10.1056/NEJMoa2001316. [PMC

doi:

article] [PubMed] [Google Scholar]

8. Hui DS, IA E, Madani TA, Ntoumi F, Kock R, Dar O. The continuing 2019-nCoV epidemic threat of novel coronaviruses to global health - the latest 2019 novel coronavirus outbreak in Wuhan, China. Int. J Infect. Dis 2020;91:264-266.

doi: 10.1016/j.ijid.2020.01.009 [PMC article] [PubMed] [Google Scholar]

9. Safi O. Scope of AYUSH Workers In Indian Health Human Resources. International Research Journal of Human Resources and Social Sciences 2018;5(4):145150.

10. Shepherd D. Homoeopathy in epidemic diseases. London: The C.W. Daniel Company Limited 1996.

11. Dudgeon RE. Hahnemann"s discovery of the prophylactic powers of belladonna in scarlet fever allopathic testimony to this prophylactic. In: Dudgeon RE, editor. Lectures on the Theory \& Practice of Homoeopathy. New Delhi: B Jain Publishers 2002, 540-4.

12. https://www.homoeopathycenter.org/treatmentepidemics-homoeopathy-history

13. Burnet F, Clark E. Influenza: a survey of the last 50 years in the light of modern work on the virus of epidemic Jama 1942;120(5):408. doi:10.1001/jama.1942.02830400082031

14. Winston J. Influenza-1918: Homeopathy To The Rescue. Spring/Summer; The New England Journal of 
Homeopathy 1998;(1):1-3.

15. Williams DA. Influenza: Brief Comments. The Journal of the American Institute of Homoeopathy, Chicago 1919;XI(9):1003-1005.

16. Baker HB. Clinical Comment on Influenza. The Journal of the American Institute of Homoeopathy, Chicago, December 1918;XI(6):682-684.

17. Pearson WA. Epidemic Influenza Treated by Homoeopathic Physicians. The Journal of the American Institute of Homoeopathy, Chicago 1919;XII(1):11-13.

18. Hui DSC, Zumla A. Severe Acute Respiratory Syndrome: historical, epidemiologic, and clinical features. Infect Dis Clin North Am. 2019;33(4):869889. doi: 10.1016/j.idc.2019.07.001

19. Zheng J, Hassan S, Alagaili AN, Alshukairi AN, Amor NMS, Mukhtar N, et al. Middle East Respiratory syndrome coronavirus seropositivity in Camel handlers and their families, Pakistan. Emerg Infect dis 2019;25(12):2307-2309. doi: 10.3201/eid2512.191169s

20. Shehata MM, Gomaa MR, Ali MA, Kayali G. Middle East respiratory syndrome coronavirus: a comprehensive review. Front Med. 2016;10(2):120136. doi: 10.1007/s11684-016-0430-6.

21. Rasmussen SA, Watson AK, Swerdlow DL. Middle East Respiratory Syndrome (MERS). Microbiol Spectr 2016;4(3). doi:10.1128/microbiolspec.EI10-0020-2016.

22. Vos LM, Bruyndonckx R, Zuithoff NPA, Little P, Oosterheert JJ, Broekhuizen BDL, et al. Lower respiratory tract infection in the community: associations between viral aetiology and illness course. Clin Microbiol Infect 2021;27(1):96-104. doi: 10.1016/j.cmi.2020.03.023

23. https://www.homoeopathy2health.com/post/safe-fromcorona

24. Robert Mathie T. Homoeopathic treatment of patients with influenza-like illness during the 2009 A/H1N1 influenza pandemic in India Elsevier 2013;102:187-92.

25. Jamadade AK, Adi BS, Reddy ESR. Single blind randomized control study on the efficacy of homoeopathic management in coronary artery disease. International journal of alternative and complementary medicine 2020, 41-45.

26. Nour Ramadan, Houssam Shaib. Middle East respiratory syndrome coronavirus (MERS-CoV) A Review Germs 2019;9(1):35-42. doi: 10.18683/germs.2019.1155

27. Arabi YM, Arifi AA, Balkhy HH, et al. Clinical course and outcomes of critically ill patients with Middle East respiratory syndrome coronavirus infection. Ann Intern Med. 2014;160(6):389-97.doi: 10.7326/M13-2486

28. Kalliantas D, Kallianta M, Karagianni CS. Homoeopathy combat against coronavirus disease (COVID-19). J Public Health (Berl.) 2020;5:1-4.doi: 10.1007/s10389-020-01305-z

29. Wang W, Tang J, Wei F. Updated understanding of the outbreak of 2019 novel coronavirus (2019-nCoV) in Wuhan. China J Med Virol. 2020;92:441-447.doi: 10.1002/jmv.25689

30. Wan Y, Shang J, Graham R, Baris RS, Li F. Receptor Recognition by the Novel Coronavirus from Wuhan: An Analysis Based on Decade-Long Structural Studies of SARS Coronavirus. J Virol. 2020;94(7):e00127-20. doi: 10.1128/JVI.00127-20.

31. Corona Ayush. 2020. Pdf [Internet]. Google Docs.
[Cite2020 May 1]. Available from:https://drive.google.com/file/d/1EiHVMrKpHL8Q JANQ_b0bBFGIVNWQ24Bj/

32. Nambison NK, Dhar Dwivedi A, Nambison SN. Pandemic COVID-19: Homoeopathic prevention and treatment experiences. Int $\mathrm{J}$ Homoeopath Sci 2020;4(2):265-270.

33. https://indianexpress.com/article/explained/debateover-a-homoeo-drug-arsenicum-album-coronavirusvaccine-6439697/

34. Kasariyans A, Sankaran R. Homoeopathy for Coronavirus COVID-19 Infection. [Internet]. NSA: Homoeopathy plus 2020;5:1-4. doi: 10.1007/s10389020-01305-zhttps://homoeopathyplus.com/wpcontent/uploads/2020/03/Homoeopathy-forCoronavirus-COVID-19

35. https://www.forbesindia.com/article/coronavirus/homoe opathy-to-fight-the-coronavirus-think-again/60135/1

36. https://www.businesstoday.in/latest/trends/herdimmunity-homoepathy-not-lockdown-best-to-fightcoronavirus-rajiv-bajaj/story/400768.html 